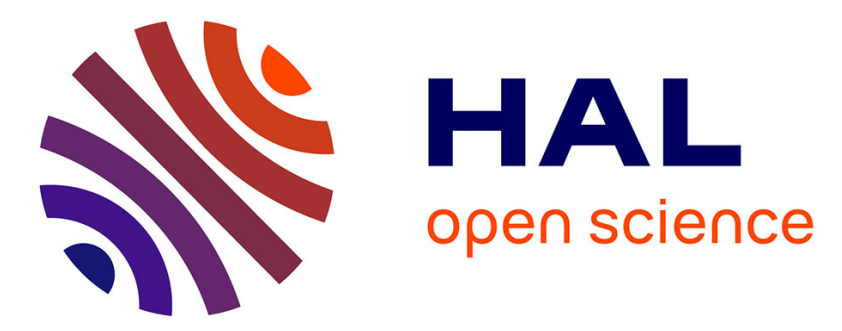

\title{
A Multi-Layer Line Search Method to Improve the Initialization of Optimization Algorithms
}

\author{
Benjamin Ivorra, Bijan Mohammadi, Angel Manuel Ramos
}

\section{To cite this version:}

Benjamin Ivorra, Bijan Mohammadi, Angel Manuel Ramos. A Multi-Layer Line Search Method to Improve the Initialization of Optimization Algorithms. European Journal of Operational Research, 2015, 247 (3), pp.711-720. 10.1016/j.ejor.2015.06.044 . hal-01206339

\section{HAL Id: hal-01206339 \\ https://hal.science/hal-01206339}

Submitted on 29 Sep 2015

HAL is a multi-disciplinary open access archive for the deposit and dissemination of scientific research documents, whether they are published or not. The documents may come from teaching and research institutions in France or abroad, or from public or private research centers.
L'archive ouverte pluridisciplinaire HAL, est destinée au dépôt et à la diffusion de documents scientifiques de niveau recherche, publiés ou non, émanant des établissements d'enseignement et de recherche français ou étrangers, des laboratoires publics ou privés. 


\title{
A Multi-Layer Line Search Method to Improve the Initialization of Optimization Algorithms
}

\author{
Benjamin Ivorra ${ }^{1, \dagger}$, Bijan Mohammadi ${ }^{2}$ and Angel Manuel Ramos ${ }^{1}$ \\ 1. Departamento de Matemática Aplicada $\&$ Instituto de Matemática Interdisciplinar, \\ Universidad Complutense de Madrid, Plaza de Ciencias, 3, 28040, Madrid, Spain \\ †Tel.: +34-91-394-4415, email: ivorra@mat.ucm.es \\ 2. Institut de Mathématiques et de Modélisation de Montpellier, Université de \\ Montpellier II, Place Eugène Bataillon, 34095, Montpellier, France
}

\begin{abstract}
We introduce a novel metaheuristic methodology to improve the initialization of a given deterministic or stochastic optimization algorithm. Our objective is to improve the performance of the considered algorithm, called core optimization algorithm, by reducing its number of cost function evaluations, by increasing its success rate and by boosting the precision of its results. In our approach, the core optimization is considered as a suboptimization problem for a multi-layer line search method. The approach is presented and implemented for various particular core optimization algorithms: Steepest Descent, Heavy-Ball, Genetic Algorithm, Differential Evolution and Controlled Random Search. We validate our methodology by considering a set of low and high dimensional benchmark problems (i.e., problems of dimension between 2 and 1000). The results are compared to those obtained with the core optimization algorithms alone and with two additional global optimization methods (Direct Tabu Search and Continuous Greedy Randomized Adaptive Search). These latter also aim at improving the initial condition for the core algorithms. The numerical results seem to indicate that our approach improves the performances of the core optimization algorithms and allows to generate algorithms more efficient than the other optimization methods studied here. A Matlab optimization package called "Global Optimization Platform" (GOP), implementing the algorithms presented here, has been developed and can be downloaded at: http://www.mat.ucm.es/momat/software.htm
\end{abstract}

Keywords: Metaheuristics; Global optimization; Multi-layer line search 
algorithms; Evolutionary algorithms; Gradient methods

\section{Introduction}

We consider a general optimization problem of the form:

$$
\min _{x \in \Omega} h_{0}(x)
$$

where $h_{0}: \Omega \rightarrow \mathbb{R}$ is the cost function, $x$ is the optimization parameter and $\Omega \subset \mathbb{R}^{N}$, with $N \in \mathbb{N}$, is the admissible space.

When solving (1) by an iterative procedure the choice of the initial condition is essential. For instance, this is the case with the gradient methods such as the Steepest Descent algorithm (SD) (Luenberger and Ye, 2008), the Newton algorithm (Polyak, 2007) or with the Heavy-Ball algorithm (HB) (Attouch et al., 2000). When $h_{0}$ has several local minima these algorithms converge to one of those depending on their initialization. However, these algorithms can still find the global optimum if the initial condition belongs to the attraction basin of the infimum. Another example where the initialization is of prime importance is with Genetics Algorithms (GA) (Goldberg, 1989; Gonalves et al., 2002) where a lack of diversity in the individuals of the initial population can result to a premature convergence to a local minimum of $h_{0}$ (Rocha and Neves, 1999).

Thus, developing methods that intend to generate suitable initial conditions is interesting in order to improve the efficiency of existing optimization methods. For a given convergence accuracy, a better initialization may lead to a reduction in the number of functional evaluations, which is particularly important when working with expensive functional evaluations as in industrial design problems (Carrasco et al., 2012, 2015; Muyl et al., 2004; Gomez et al., 2011; Ivorra et al., 2006, 2009, 2013, 2014).

The idea of improving optimization algorithms by choosing a suitable initialization is widely present in the literature. For instance, the Direct Tabu Search algorithm (DTS) (Hedar and Fukushima, 2006; Lamghari and Dimitrakopoulos, 2012) and the Tunneling algorithm (Gomez and Levy, 1982; Levy and Gomez., 1985), are based on a modification of the functional by the addition of penalty terms to avoid the algorithm to revisit previously explored regions. Other techniques, like the Greedy Randomized Adaptive Search Procedure (GRASP) (Hirsch et al., 2010; Mart et al., 2015) or the 
Universal Evolutionary Global Optimizer (Redondo et al., 2009)are based on the construction of a greedy solution combined with a local search step.

Another technique consists in coupling line search methods (Luenberger and Ye, 2008; Vieira and Lisboa, 2014) with another optimization algorithm. For instance, in Gardeux et al. (2011) the authors propose an optimization method, called EM323, well suited for the solution of high-dimensional continuous non-linear optimization problems. The algorithm EM323 consists in combining the Enhanced Unidirectional Search method (Gardeux et al., 2009) with the 3-2-3 line search procedure (Glover, 2010). Another example can be found in Grosan and Abraham (2007), in the context of MultiObjective optimization problems. The authors develop a method combining several line search algorithms: one for determining a first point in the Pareto front and another one for exploring the front.

In this work, we propose a novel metaheuristic technique also based on line search methods to dynamically improve the initialization of a given optimization method. The paper is organized as follows. In Section 2 we reformulate problem (1) as a sub-optimization problem where the initial condition of the considered optimization algorithm is the optimization parameter. This new problem is solved by considering an original multi-layer semi-deterministic line search algorithm. In Section 3, we focus on the implementation of our approach by considering two families of optimization algorithms: descent methods (in particular, SD and HB) and Evolutionary Algorithms (in particular, GAs, Controlled Random Search algorithms (CRS) (Price, 1983) and Differential Evolution algorithms (DE) (Price et al., 2005)). In Section 4, we validate our approach by considering various test cases in both low (Floudas and Pardalos, 1999) and high (Li et al., 2013) dimensions. The results are then compared with those given by the following optimization algorithms: SD, HB, DTS, Continuous GRASP (CGR), CRS, DE and GA.

\section{General optimization method}

We consider an optimization algorithm $A_{0}: V \rightarrow \Omega$, called core optimization algorithm (COA), to solve problem (1). Here, $V$ is the space where we can choose the initial condition for $A_{0}$ (various examples are given in Section 3 , for simplicity we can consider $V=\Omega$ ). The other optimization parameters of $A_{0}$ (such as the stopping criterion, the number of iterations, etc.) are 
fixed by the user. We omit them in the presentation in order to simplify the notations.

We assume the existence of $v \in V$ such that, for a given precision $\epsilon \geq 0$, $h_{0}\left(A_{0}(v)\right)-\min _{x \in \Omega} h_{0}(x)<\epsilon$. Thus, solving problem (1) with algorithm COA means:

$$
\text { Find } v \in V \text { such that } A_{0}(v) \in \operatorname{argmin}_{x \in \Omega} h_{0}(x) .
$$

In order to solve problem (2), we propose to use a multi-layer semideterministic algorithm (called in the sequel the Multi-Layer Algorithm and denoted by MLA) based on line search methods (see, for instance, Luenberger and Ye (2008); Vieira and Lisboa (2014); Mohammadi and Saïac (2003)).

More precisely, we introduce $h_{1}: V \rightarrow \mathbb{R}$ as:

$$
h_{1}(v)=h_{0}\left(A_{0}(v)\right) \text {. }
$$

Thus, problem (2) can be rewritten as

$$
\text { Find } v \in V \text { such that } v \in \operatorname{argmin}_{w \in V} h_{1}(w) \text {. }
$$

A geometrical representation of $h_{1}($.$) in one dimension is shown in Figure$ 1 for a situation where the COA is the SD applied with 10000 iterations, $\Omega=V=[-10,6]$ and $h_{0}(x)=\frac{1}{2} \cos (2 x)+\sin \left(\frac{1}{3} x\right)+1.57$. We see that $h_{1}($.$) is discontinuous with plateaus. Indeed, the same solution is reached$ by the algorithm starting from any of the points of the same attraction basin. Furthermore, $h_{1}($.$) is discontinuous where the functional reaches a$ local maximum. One way to minimize such kind of functionals in the one dimensional case is to consider line search optimization methods (such as the secant or the dichotomy methods, see Mohammadi and Saïac (2003)).

Thus, in order to solve problem (4), we introduce the algorithm $A_{1}: V \rightarrow$ $V$ which, for any $v_{1} \in V$, returns $A_{1}\left(v_{1}\right) \in V$ after the following steps:

Step 1- Choose $v_{2}$ randomly in $V$.

Step 2- Find $v \in \operatorname{argmin}_{w \in \mathcal{O}\left(v_{1}, v_{2}\right)} h_{1}(w)$, where $\mathcal{O}\left(v_{1}, v_{2}\right)=\left\{v_{1}+t\left(v_{2}-\right.\right.$ $\left.\left.v_{1}\right), t \in \mathbb{R}\right\} \cap V$, using a line search method.

Step 3- Return $v$. 


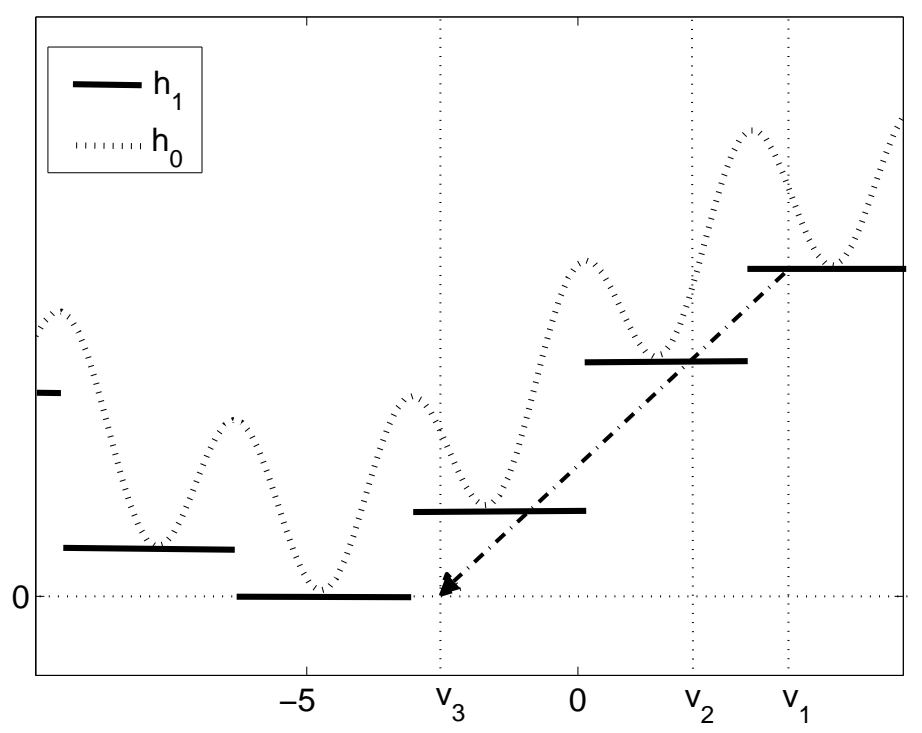

Figure 1: (Dotted line) Graphical representation of $h_{0}(x)=\frac{1}{2} \cos (2 x)+\sin \left(\frac{1}{3} x\right)+1.57$, for $x \in \Omega=V=[-10,6]$. (Continuous line) Graphical representation of $h_{1}($.$) when the$ SD is used as COA with 10000 iterations. (Slash-dotted line) Graphical representation of one execution of the algorithm $A_{1}\left(v_{1}\right)$, described in Section 3.1.1, when $v_{1}$ is given and $t_{1}=1 . v_{2}$ is generated randomly in $[-10,6]$ in the first Step of the algorithm. $v_{3}$ is built by the secant method performed in Step 2.2. $v_{3}$ (the best initial condition) is returned as the output in Step 3, since $h_{1}\left(v_{3}\right)$ is lower than $h_{1}\left(v_{1}\right)$ and $h_{1}\left(v_{2}\right)$. 
The user may choose of the line search minimization algorithm in $A_{1}$.

This construction can be pursued looking for an optimal initialization for $A_{1}$. This can be done adding an external layer to algorithm $A_{1}$ and introducing $h_{2}: V \rightarrow \mathbb{R}$ defined by

$$
h_{2}(v)=h_{1}\left(A_{1}(v)\right)
$$

and considering the following problem:

$$
\text { Find } v \in V \text { such that } v \in \operatorname{argmin}_{w \in V} h_{2}(w) \text {. }
$$

To solve problem (6), we use the two-layers algorithm $A_{2}: V \rightarrow V$ that, for each $v_{1} \in V$, returns $A_{2}\left(v_{1}\right) \in V$ given by

Step 1- Choose $v_{2}$ randomly in $V$.

Step 2- Find $v \in \operatorname{argmin}_{w \in \mathcal{O}\left(v_{1}, v_{2}\right)} h_{2}(w)$ using a line search method.

Step 3- Return $v$.

As previously, the user may choose the line search minimization algorithm in $A_{2}$. Due to the fact that the line search direction $\mathcal{O}\left(v_{1}, v_{2}\right)$ in $A_{1}$ is constructed randomly, the algorithm $A_{2}$ performs a multi-directional search of the solution of problem (2).

This construction can be pursued recursively defining

$$
h_{i}(v)=h_{i-1}\left(A_{i-1}(v)\right), \quad \text { for } \quad i \in \mathbb{N},
$$

and considering the problem

$$
\text { Find } v \in V \text { such that } v \in \operatorname{argmin}_{w \in V} h_{i}(w) \text {. }
$$

Problem (8) is solved by using the $i$-layers algorithm $A_{i}: V \rightarrow V$ that, for each $v_{1} \in V$, returns $A_{i}\left(v_{1}\right)$ given by

Step 1- Choose $v_{2}$ randomly in $V$.

Step 2- Find $v \in \operatorname{argmin}_{w \in \mathcal{O}\left(v_{1}, v_{2}\right)} h_{i}(w)$ using a line search method.

Step 3- Return $v$.

In practice, as specified in Section 4 , we run $A_{i}$ with a suitable stopping criterion and with $v_{1} \in V$ arbitrary (or $v_{1} \in V$ a good initial guess, if available). 
The choice of the random technique used to generate $v_{2}$ in Step 1 of $A_{i}$ is important and depends on $h_{0}$. For instance, if we know that $h_{0}$ has several local minima in $\Omega$ with a small attraction basins, it seems appropriate to generate $v_{2}$ in a small neighborhood of $v_{1}$.

Also, the line search minimization algorithm used in Step 2 of $A_{i}$ depends on the properties of $h_{0}$, as discussed in Section 3 .

\section{MLA implementation}

In this Section, we present various MLA implementations, considering different COAs, in the particular case where $h_{0}$ is a non negative function (or, equivalently, greater than a known real number). This specific situation often occurs in industrial problems (see, for instance, Ivorra et al. (2007, 2009, 2006, 2014)).

In particular, we consider two classes of implementations of the MLA associated with two kinds of COAs: gradient and evolutionary algorithms.

\section{1. $\boldsymbol{M L A}$ implementation with descent methods}

We consider a core algorithm $A_{0}$ that comes from the discretization of the following initial value problem (Attouch et al., 2000):

$$
\left\{\begin{array}{c}
\eta \frac{\mathrm{d}^{2} x}{\mathrm{~d} t^{2}}(t)+M(x(t), t) \frac{\mathrm{d} x}{\mathrm{~d} t}(t)=-d(x(t)), \quad t \geq 0 \\
x(0)=x_{0}, \quad \frac{\mathrm{d} x}{\mathrm{~d} t}(0)=x_{t, 0},
\end{array}\right.
$$

where $t$ is a fictitious time, $\eta \in \mathbb{R}, M: \Omega \times \mathbb{R} \rightarrow M_{N \times N}$ (with $M_{N \times N}$ denoting the set of matrix $N \times N)$ and $d: \Omega \rightarrow \mathbb{R}^{N}$ is a function giving a descent direction. For instance:

- Assuming $h_{0} \in C^{1}(\Omega, \mathbb{R})$, when $\eta=0, d=\nabla h_{0}$ and $M(x, t)=\mathrm{Id}$ (the identity operator) for all $(x, t) \in \Omega \times \mathbb{R}$, we recover, considering a suitable discretization, the SD method (Luenberger and Ye, 2008).

- Assuming $h_{0} \in C^{2}(\Omega, \mathbb{R})$, when $d=\nabla h_{0}$ and $M(x, t)=\operatorname{Id}$ for all $(x, t) \in$ $\Omega \times \mathbb{R}$, we recover, considering an adequate discretization, the HB method (Attouch et al., 2000). This algorithm presents an exploratory character in the optimization process, in comparison to the case $\eta=0$, allowing to escape from attraction basins. 
According to the previous notation, we denote by $A_{0}\left(x_{0}, x_{t, 0}\right)$ the solution returned by the COA starting from $x_{0} \in \Omega$ with an initial "velocity" $x_{t, 0} \in$ $\mathbb{R}^{N}$ and the set of remaining algorithm parameters fixed by the user.

We consider two possible choices for the initial condition of the COA (either $x_{0}$ or $x_{t, 0}$ ) leading to two different formulations of problem (2). In the following, we propose the corresponding MLAs for solving each case.

\subsubsection{Considering $x_{0}$ as the initial condition}

We consider the following formulation of problem (2):

$$
\text { Find } x_{0} \in V=\Omega \text { such that } A_{0}\left(x_{0}, x_{t, 0}\right) \in \operatorname{argmin}_{w \in \Omega} h_{0}(w) \text {, }
$$

where $x_{t, 0} \in \mathbb{R}^{N}$ is fixed. We note that any $x_{0} \in \operatorname{argmin}_{x \in \Omega} h_{0}(x)$ is a solution of (10).

To solve problem (10), we consider a particular implementation of the algorithms $A_{i}, i \in \mathbb{N}$, introduced previously. For $i \in \mathbb{N}, A_{i}\left(v_{1}\right)$ is applied with a secant method in order to perform the line search as following:

Step 1- Choose $v_{2} \in \Omega$ randomly.

Step 2- For $l$ from 1 to $t_{i} \in \mathbb{N}$ :

Step 2.1- If $h_{i}\left(v_{l}\right)=h_{i}\left(v_{l+1}\right)$ go to Step 3

Step 2.2- Set $v_{l+2}=\operatorname{proj}_{\Omega}\left(v_{l+1}-h_{i}\left(v_{l+1}\right) \frac{v_{l+1}-v_{l}}{h_{i}\left(v_{l+1}\right)-h_{i}\left(v_{l}\right)}\right)$,

where $\operatorname{proj}_{\Omega}: \mathbb{R}^{N} \rightarrow \Omega$ is a projection algorithm over $\Omega$ defined by the user.

Step 3- Return the output: $\operatorname{argmin}\left\{h_{i}\left(v_{m}\right), m=1, \ldots, t_{i}\right\}$.

In the previous algorithm, the values $i$ and $t_{i}$ depend on the desired computational complexity. In Section 4, we check the efficiency of those algorithms for various set of values.

A geometrical representation of one execution of algorithm $A_{1}$ in one dimension is shown in Figure 1.

From a theoretical point of view, as the secant method is adapted to find the zeros of a function (Mohammadi and Saïac, 2003), those algorithms perform better if the minimum value of $h_{0}$, denoted by $h_{0}^{*} \in \mathbb{R}$, is known (for instance, in some inverse problems, see Ivorra et al. (2014)). Indeed, we can assume that $h_{0}^{*}=0$ (we minimize $h_{0}-h_{0}^{*}$ instead of $h_{0}$ ) and thus $A_{i}$ intends to find the zero of $h_{i}$ (see Ivorra (2006) for more details). However, in practice (see experiments presented in Section 4), if the only information 
available is that $h_{0}$ is greater than a known real number $h_{l} \in \mathbb{R}$, we consider $h_{0}-h_{l}$ instead of $h_{0}$ and this algorithm still gives good numerical results. This efficiency can be in part explained by the structure of the secant iteration that gives a quick information about the behavior of $h_{0}$. If there is a significant evolution of the cost function value between $v_{l+1}$ and $v_{l}$, the secant iteration generates $v_{l+2}$ close to $v_{l+1}$ (because the slope of the straight line passing trough the points $\left(v_{l+1}, h_{0}\left(v_{l+1}\right)\right)$ and $\left(v_{l+2}, h_{0}\left(v_{l+2}\right)\right)$ is steep) performing a refined search around $v_{l+1}$. Otherwise, the secant method generates $v_{l+2}$ far from $v_{l+1}$ allowing to explore a new region.

Remark 1. Although this case is not considered in this paper, if no information about a lower bound value of $h_{0}$ is available we could consider other $\boldsymbol{M L A}$ implementations. For instance, we can replace the secant method used in $\boldsymbol{S t e p} 2.2$ by the $\boldsymbol{S D}$ iteration starting from $v_{l+1}$ and using $-\frac{v_{l+1}-v_{l}}{h_{i}\left(v_{l+1}\right)-h_{i}\left(v_{l}\right)}$ as the descent direction. This new step reads:

Step 2.2- Set $v_{l+2}=\operatorname{proj}_{\Omega}\left(v_{l+1}-\rho \frac{v_{l+1}-v_{l}}{h_{i}\left(v_{l+1}\right)-h_{i}\left(v_{l}\right)}\right)$, where $\operatorname{proj}_{\Omega}: \mathbb{R}^{N} \rightarrow$ $\Omega$, is a projection algorithm over $\Omega$ defined by the user and $\rho \in \mathbb{R}$ is obtained by solving numerically $\min _{\rho \in \mathbb{R}^{+}} h_{0}\left(\operatorname{proj}_{\Omega}\left(v_{l+1}-\rho \frac{v_{l+1}-v_{l}}{h_{i}\left(v_{l+1}\right)-h_{i}\left(v_{l}\right)}\right)\right)$ by using a dichotomy method.

\subsubsection{Considering $x_{t, 0}$ as the initial condition}

We consider the following formulation of problem (2):

Find $x_{t, 0} \in V=\mathbb{R}^{N}$ such that $A_{0}\left(x_{0}, x_{t, 0}\right) \in \operatorname{argmin}_{w \in \Omega} h_{0}(w)$,

where $x_{0} \in \Omega$ is fixed. In this case, under convenient hypotheses, we can prove the existence of $x_{t, 0} \in \mathbb{R}^{N}$ such that the solution of problem (11) can be approximated numerically, as stated in the following theorem:

Theorem 1. Let $h_{0} \in C^{2}\left(\mathbb{R}^{N}, \mathbb{R}\right)$ having a minimum at $x_{m} \in \mathbb{R}^{N}$. We assume that its gradient, denoted by $\nabla h_{0}($.$) , is Lipschitz continuous. Thus,$ for every $\left(x_{0}, \epsilon\right) \in \mathbb{R}^{N} \times \mathbb{R}^{+}$, there exists $\left(\sigma, \tau_{b}\right) \in \mathbb{R}^{N} \times \mathbb{R}^{+}$such that the solution of the following dynamical system

$$
\left\{\begin{array}{l}
\eta \frac{\mathrm{d}^{2} x}{\mathrm{~d} t^{2}}(t)+\frac{\mathrm{d} x}{\mathrm{~d} t}(t)=-\nabla h_{0}(x(t)), \quad t \geq 0, \\
x(0)=x_{0}, \quad \frac{\mathrm{d} x}{\mathrm{~d} t}(0)=\sigma,
\end{array}\right.
$$

with $\eta \in \mathbb{R}$, passes at time $\tau_{b}$ into the ball of center $x_{m}$ and radius $\epsilon$, denoted by $B_{\epsilon}\left(x_{m}\right)$. 
Proof : We assume $x_{0} \neq x_{m}$ (as $x_{0}=x_{m}$ is a trivial case). Let $\delta \geq 0$, we consider the initial value problem

$$
\left\{\begin{array}{l}
\eta \frac{\mathrm{d}^{2} y_{\delta}}{\mathrm{d} t^{2}}(t)+\delta \frac{\mathrm{d} y_{\delta}}{\mathrm{d} t}(t)(t)=-\delta^{2} \nabla h_{0}\left(y_{\delta}(t)\right), \quad t \geq 0, \\
y_{\delta}(0)=x_{0}, \quad \frac{\mathrm{d} y_{\delta}}{\mathrm{d} t}(t)(0)=\varrho\left(x_{m}-x_{0}\right),
\end{array}\right.
$$

with $\varrho \in \mathbb{R}^{+} \backslash\{0\}$. Let us show that $y_{\delta}$ passes at some time into the ball $B_{\epsilon}\left(x_{m}\right)$ :

- If $\delta=0$, we obtain the following system

$$
\left\{\begin{array}{l}
\eta \frac{\mathrm{d}^{2} y_{0}}{\mathrm{~d} t^{2}}(t)=0, \quad t \geq 0 \\
y_{0}(0)=x_{0}, \quad \frac{\mathrm{d} y_{0}}{\mathrm{~d} t}(0)=\varrho\left(x_{m}-x_{0}\right)
\end{array}\right.
$$

System (14) describes a straight line of origin $x_{0}$ and passing at some time $\tau_{\varrho} \in \mathbb{R}^{+}$by the point $x_{m}$ (i.e., $y_{0}\left(\tau_{\varrho}\right)=x_{m}$ ).

- If $\delta \neq 0$, System (13) can be rewritten as

$$
\frac{\mathrm{d} w}{\mathrm{~d} t}(t)=\left(\begin{array}{c}
\frac{\mathrm{d} y_{\delta}}{\mathrm{d} t}(t) \\
-\delta \frac{\mathrm{d} y_{\delta}}{\mathrm{d} t}(t)-\delta^{2} \nabla h_{0}\left(y_{\delta}(t)\right)
\end{array}\right)=f(t, w(t), \delta),
$$

with $w(t)=\left(y_{\delta}(t), \eta \frac{\mathrm{d} y_{\delta}}{\mathrm{d} t}(t)\right)$ and $f$ continuous in $t$ and in $\delta$ and Lipschitz continuous in $w(t)$ (Attouch et al., 2000). Then, applying the Cauchy-Lipschitz theorem (see, for instance, Verhulst (1996)) and the Continuity Theorem 3.4 found in Hale (2009), we obtain that

$$
\lim _{\delta \rightarrow 0}\left|y_{\delta}\left(\tau_{\varrho}\right)-y_{0}\left(\tau_{\varrho}\right)\right|=0
$$

Thus, for every $\epsilon \in \mathbb{R}^{+} \backslash\{0\}$, there exists $\delta_{\epsilon} \in \mathbb{R}^{+}$such that for every $\delta \leq \delta_{\epsilon}$

$$
\left|y_{\delta}\left(\tau_{\varrho}\right)-x_{m}\right|<\epsilon
$$


Let $\epsilon \in \mathbb{R}^{+} \backslash\{0\}$. We consider the change of variable given by $s=\delta_{\epsilon} t$ and $x(s)=y_{\delta_{\epsilon}}\left(\frac{s}{\delta_{\epsilon}}\right)$. Then, System (13) becomes

$$
\left\{\begin{array}{l}
\eta \frac{\mathrm{d}^{2} x}{\mathrm{~d} s^{2}}(s)+\frac{\mathrm{d} x}{\mathrm{~d} s}(s)=-\nabla h_{0}(x(s)), \quad s \geq 0, \\
x(0)=x_{0}, \quad \frac{\mathrm{d} x}{\mathrm{~d} s}(0)=\frac{\varrho}{\delta_{\epsilon}}\left(x_{m}-x_{0}\right) .
\end{array}\right.
$$

Let $\tau_{b}=\delta_{\epsilon} \tau_{\varrho}$. Under this assumption, $x\left(\tau_{b}\right)=y_{\delta_{\epsilon}}\left(\tau_{\varrho}\right)$. Thus, due to (16), $\left|x\left(\tau_{b}\right)-x_{m}\right|<\epsilon$. We have found $\sigma=\frac{\varrho}{\delta_{\epsilon}}\left(x_{m}-x_{0}\right) \in \mathbb{R}^{N}$ and $\tau_{b} \in \mathbb{R}^{+}$such that the solution of System (12) passes at time $\tau_{b}$ into the ball $B_{\epsilon}\left(x_{m}\right)$.

In order to determine a solution of problem (11), we can consider, for example, the same implementation of algorithms $A_{i}$, with $i \in \mathbb{N}$, as the one introduced in Section 3.1.1.

\section{2. $\boldsymbol{M L A}$ implementation with Evolutionary Algorithms}

\subsubsection{General framework}

Evolutionary Algorithms (EA) are population-based metaheuristic optimization algorithms which try to solve problems similar to (2) (Ashlock, 2010). From a general point of view, they start from a finite set of points in the search space $\Omega$, called initial population, and intend to improve the value of the considered cost function by applying processes based on an analogy with the Darwinian evolution of species. For instance, we can cite some classical EAs (considered in the experiments presented in Section 4): the Genetic (Goldberg, 1989), the Controlled Random Search (Price, 1983) and the Differential Evolution (Price et al., 2005) algorithms. The EAs have many advantages, as for example: they generally do not require sensitivity computation, they can solve complex optimization problems (e.g., with high dimensional search space or function with various with local minima), and they are intrinsically parallel. However, they also have some important drawbacks as: slower convergence and lower accuracy than other methods (such as gradient algorithms (Ashlock, 2010)).

We denote by $X^{0}=\left\{x_{j}^{0} \in \Omega, j=1, \ldots, N_{p}\right\}$, with $N_{p} \in \mathbb{N}$, the set corresponding to the initial population of the considered EA. All other parameters of the EA are fixed by the user. In this case, problem (2) can be rewritten as:

$$
\text { Find } X^{0} \in V=\Omega^{N_{p}} \text { such that } A_{0}\left(X^{0}\right) \in \operatorname{argmin}_{w \in \Omega} h_{0}(w) \text {. }
$$


In the following, we propose a version of the MLA used to solve problem (18). This MLA is illustrated by considering a particular Genetic Algorithm (GA), validated previously in Ivorra (2006); Ivorra et al. $(2006,2014)$, as the COA. We note that the proposed algorithm can be easily extended to any other EA, as illustrated in Section 4 with the use of a Controlled Random Search algorithm (CRS) and a Differential Evolution algorithm (DE) as COAs.

As it is out of the scope in this paper, we do not describe the considered CRS and DE implementations, which are deeply detailed in Hendrix et al. (2001) and Storn and Price (1997), respectively, but we give in Section 3.2.2 the implementation that we use for the $\mathbf{G A}$.

\subsection{2. $\boldsymbol{M L A}$ implementation with $G A$}

We first describe the GA considered here:

- Step 1- Inputs: User must define the parameters $N_{p} \in \mathbb{N}, N_{g} \in \mathbb{N}$, $p_{m} \in[0,1], p_{c} \in[0,1], \epsilon \in \mathbb{R}$ and the initial population $X^{0} \in \Omega^{N_{p}}$. The meaning of those parameters is clarified later in the following steps.

- Step 2- Generating new populations: Starting from $X^{0}$, we recursively create $N_{g}$ new populations by applying four stochastic processes: 'selection', 'crossover', 'mutation' and 'elitism', which are described in Steps 3.1, 3.2, 3.3 and 3.4, respectively.

More precisely, let $X^{i}=\left\{x_{j}^{i} \in \Omega, j=1, \ldots, N_{p}\right\}$, with $i=1, \ldots, N_{g}-1$, denotes the population at iteration $i$. Then, using the $\left(N_{p}, N\right)$-real valued matrix

$$
X^{i}=\left[\begin{array}{c}
x_{1}^{i} \\
\vdots \\
x_{N_{p}}^{i}
\end{array}\right]=\left[\begin{array}{ccc}
x_{1}^{i}(1) & \ldots & x_{1}^{i}(N) \\
\vdots & \ddots & \vdots \\
x_{N_{p}}^{i}(1) & \ldots & x_{N_{p}}^{i}(N)
\end{array}\right]
$$

with $x_{j}^{i}=\left(x_{j}^{i}(1), \ldots, x_{j}^{i}(N)\right) \in \Omega, X^{i+1}$ is obtained by considering:

$$
X^{i+1}=\left(I_{N}-\mathcal{E}^{i}\right)\left(\mathcal{C}^{i} \mathcal{S}^{i} X^{i}+\mathcal{M}^{i}\right)+\mathcal{E}^{i} X^{i}
$$

where matrices $\mathcal{S}^{i}, \mathcal{C}^{i}, \mathcal{M}^{i}, \mathcal{E}^{i}$ and $I_{N}$ are described below.

- Step 2.1- Selection: We randomly select $N_{p}$ individuals from $X^{i}$ with eventual repetitions. Each individual $x_{j}^{i} \in X^{i}$, with $j=$ 
$1, \ldots, N_{p}$, has a probability to be selected in this process which is given by $J^{-1}\left(x_{j}^{i}\right) / \sum_{k=1}^{N_{p}} J^{-1}\left(x_{k}^{i}\right)$. This step can be summarized as

$$
X^{i+1,1}=\mathcal{S}^{i} X^{i}
$$

where $\mathcal{S}^{i}$ is a $\left(N_{p}, N_{p}\right)$-matrix with $\mathcal{S}_{j, k}^{i}=1$ if the $k$-th individual of $X^{i}$ is the $j$-th selected individual and $\mathcal{S}_{j, k}^{i}=0$ otherwise.

- Step 2.2- Crossover: For each pair of consecutive individuals (rows) $2 j-1$ and $2 j$ in $X^{i+1,1}$, with $1 \leq j \leq$ floor $\left(N_{p} / 2\right.$ ) (where floor $(X)$ is the nearest integer lower or equal than $X$ ), we determine, with a probability $p_{c}$, if those rows exchange data or if they are directly copied into an intermediate population denoted by $X^{i+1,2}$. Mathematically, this step can be written as

$$
X^{i+1,2}=\mathcal{C}^{i} X^{i+1,1}
$$

where $\mathcal{C}^{i}$ is a real-valued $\left(N_{p}, N_{p}\right)$-matrix. The coefficients of the $(2 j-1)$-th and $2 j$-th rows of $\mathcal{C}^{i}$, with $1 \leq j \leq$ floor $\left(N_{p} / 2\right)$, are given by

$\mathcal{C}_{2 j-1,2 j-1}^{i}=\lambda_{1}, \quad \mathcal{C}_{2 j-1,2 j}^{i}=1-\lambda_{1}, \quad \mathcal{C}_{2 j, 2 j}^{i}=\lambda_{2}, \quad \mathcal{C}_{2 j, 2 j-1}^{i}=1-\lambda_{2}$

where $\lambda_{1}=\lambda_{2}=1$, with a probability $1-p_{c}$, or $\lambda_{1}$ and $\lambda_{2}$ are randomly chosen in ]0,1[, considering a uniform distribution, in other case. Other coefficients of $\mathcal{C}^{i}$ are set to 0 . If $N_{p}$ is odd then we also set $\mathcal{C}_{N_{p}, N_{p}}^{i}=1$ and then the $N_{p}$-th row of $X^{i+1,1}$ is directly copied in $X^{i+1,2}$.

- Step 2.3- Mutation: We decide, with a probability $p_{m}$, if each row of $X^{i+1,2}$ is randomly perturbed or not. This step is defined by

$$
X^{i+1,3}=X^{i+1,2}+\mathcal{M}^{i},
$$

where $\mathcal{M}^{i}$ is a real-valued $\left(N_{p}, N\right)$-matrix where the $j$-th row, $j=1, \ldots, N_{p}$, is equal to 0 , with a probability $1-p_{m}$, or a random vector $m_{j} \in \mathbb{R}^{N}$, generated considering a uniform distribution in the subset of $\mathbb{R}^{N}$ such that $x_{j}^{i+1,2}+m_{j} \in \Omega$, otherwise.

- Step 2.4- Elitism: Let $x_{b}^{i}$, where $b \in 1, \ldots, N_{p}$, be the individual in $X^{i}$ with the lowest value of $h_{0}$ (or, if there exists various, one of 
those individuals selected randomly with a uniform distribution). If $x_{b}^{i}$ has a lower $h_{0}$ value than all the individuals in $X^{i+1,3}$, it is directly copied at the $b$-th row of $X^{i+1}$. This step can be formalized as

$$
X^{i+1}=\left(I_{N}-\mathcal{E}^{i}\right)\left(X^{i+1,3}\right)+\mathcal{E}^{i} X^{i},
$$

where $I_{N}$ is the identity matrix of size $N$ and $\mathcal{E}^{i}$ is a real-valued $\left(N_{p}, N_{p}\right)$-matrix such that $\mathcal{E}^{i}(b, b)=1$ if $x_{b}^{i}$ has a lower $h_{0}$ value than all the individuals in $X^{i+1,3}$ and 0 otherwise, $\mathcal{E}^{i}=0$ elsewhere.

- Step 3- Output: After $N_{g}$ iterations or if the stopping criterion associated to $\epsilon \in \mathbb{R}$ is satisfied for at least one individual in $X^{i+1}$, the GA stops and returns an output solution denoted by

$$
\begin{aligned}
& A_{0}\left(X^{0}\right)=\operatorname{argmin}\left\{h_{0}\left(x_{j}^{i}\right) / x_{j}^{i} \text { is the } j \text {-th row of } X^{i},\right. \\
& \left.\qquad i=1, \ldots, N_{g}, j=1, \ldots, N_{p}\right\} .
\end{aligned}
$$

Remark 2. As a fine convergence is generally difficult to achieve with $\boldsymbol{G A s}$, it is recommended when it is possible, to complete the $\boldsymbol{G A}$ iterations with a descent method (Muyl et al., 2004).

The solution of problem (18) may be determined, for instance, by using the algorithms $A_{i}$ (with $i=1,2, \ldots$ ) presented in Section 3.1.1. However, previous studies (see Ivorra (2006); Ivorra et al. (2006)) show that the following variation of $A_{i}$ (with $i=0,1,2,3, \ldots$ ), denoted by $B_{i}$, is better suited to the GA case. Let $X_{1}^{0}=\left\{x_{1, j}^{0} \in \Omega, j=1, \ldots, N_{p}\right\}$ and $B_{0}\left(X^{0}\right)=A_{0}\left(X^{0}\right)$, then, for $i>0, B_{i}\left(X_{1}^{0}\right)$ reads:

Step 1- For $l$ from 1 to $t_{i} \in \mathbb{N}$ :

Step 1.1- Set $o_{l}=B_{i-1}\left(X_{l}^{0}\right)$.

Step 1.2- We construct $X_{l+1}^{0}=\left\{x_{l+1, j}^{0} \in \Omega, j=1, \ldots, N_{p}\right\}$ as following: $\forall j \in\left\{1, \ldots, N_{p}\right\}$, if $h_{0}\left(o_{l}\right)=h_{0}\left(x_{l, j}^{0}\right)$ set $x_{l+1, j}^{0}=x_{l, j}^{0}$ else set $x_{l+1, j}^{0}=\operatorname{proj}_{\Omega}\left(x_{l, j}^{0}-h_{0}\left(o_{l}\right) \frac{o_{l}-x_{l, j}^{0}}{h_{0}\left(o_{l}\right)-h_{0}\left(x_{l, j}^{0}\right)}\right)$ where $\operatorname{proj}_{\Omega}: \mathbb{R}^{N} \rightarrow \Omega$ is a projection operator over $\Omega$ defined by the user.

Step 2- Return the output: $\operatorname{argmin}\left\{h_{i}\left(o_{m}\right), m=1, \ldots, t_{i}\right\}$ 
As previously, the values of $i$ and $t_{i}$ depend on the desired computational complexity.

This version of the algorithm intends to improve, individual by individual, the initial population of $B_{i-1}$. More precisely, for each individual in the initial population:

- If there is a significant evolution of the cost function between this individual and the best element found by $B_{i-1}$, the secant method used in Step 1.2 generates, in the optimized initial population $X_{l+1}^{0}$, a new individual close to $o_{l}$ that performs a refined search near the actual solution.

- Otherwise, the secant method creates a new individual in $X_{l+1}^{0}$ far from $o_{l}$, to expand the exploration of the admissible space.

Numerical experiments in Section 4 seem to indicate that considering algorithms $B_{i}$, with $i>0$, reduces the computational complexity of GAs. In particular, this allows to reduce both parameters $N_{p}$ and $N_{g}$ in GAs. We will also analyze the application of algorithms $B_{i}$ with $\mathbf{C R S}$ and DE as the COA.

\section{Numerical experiments}

In order to check the efficiency of the MLAs presented in Section 3, we consider two sets of benchmark problems. The first set, described in Section 4.1, consists in low dimensional (i.e., dimension lower than 10) problems (Floudas and Pardalos, 1999). The second set, detailed in Section 4.2, focuses on high dimensional problems (with dimension from 50 to 1000) (Li et al., 2013). Our objective is to see how MLAs improve the efficiency of several COAs and to compare them with other metaheuristic methods.

\subsection{Low dimensional benchmark problems}

\subsubsection{Considered benchmark problems}

We consider the following set of box-constrained benchmark optimization problems: Branin (denoted by Bra), Eason (Eas), Goldstein-Price (G-P), Shubert (Shu), Hartmann with 3 (Hm3) and 6 (Hm6) variables, Rosenbrock with 2 (Rb2), 5 (Rb5) and 10 (Rb10) variables, Shekel with 4 variables and $m=5$ (Sk5), 7 (Sk7)and 10 (Sk10), and Zakharov with 5 (Za5) and $10(\mathbf{Z a 1 0})$ variables. A complete description of those problems with 
the considered values of the box restrictions can be found in Floudas and Pardalos (1999). These problems well feature the difficulties of optimization problems and are frequently used to validate optimization algorithms (Hedar and Fukushima, 2006; Hirsch et al., 2010).

\subsubsection{Considered algorithms and parameters}

To solve numerically the problems introduced in Section 4.1.1, we consider the following MLAs:

- When the SD is the COA, we use the MLA implementation presented in Section 3.1.1. We apply this method with different number of layers $i: i=1$ (the algorithm is then denoted by SMA1, as SD Multi-Layer Algorithm 1-Layer), $i=2$ (SMA2) and $i=3$ (SMA3). We set $t_{0}=10$ and $t_{1}=1000$ for SMA1; $t_{0}=t_{1}=10$ and $t_{2}=1000$ for SMA2; and $t_{0}=t_{1}=t_{2}=10$ and $t_{3}=1000$ for SMA3. The descent step size $\rho$ is determined using 10 iterations of a dichotomy method starting from $\rho_{0}=1$ (Mohammadi and Saïac, 2003). Those parameters have been determined in Debiane et al. (2006); Ivorra (2006); Ivorra et al. (2006).

- When the HB is used as the COA, we use the two-layers algorithm $A_{2}$, described in Sections 3.1.1 and 3.1.2, with $\eta=0.1, t_{0}=t_{1}=10$ and $t_{2}=1000$. The velocity $x_{t, 0}$ is the initial condition to be optimized. $x_{0}$ is chosen randomly in $\Omega$. This algorithm is denoted by HMA (HB Multi-Layer Algorithm). Those specific parameters have been proposed in Ivorra (2006).

- With GA as the COA, we use the algorithm $B_{2}$ introduced in Section 3.2 with $t_{1}=10$ and $t_{2}=1000$. This algorithm is denoted by GMA (Genetic Multi-Layer Algorithm). The GA parameters are set to: $N_{p}=10, N_{g}=10, p_{c}=0.55, p_{m}=0.5$. Those parameters have been considered in Gomez et al. (2011); Ivorra (2006); Ivorra et al. (2013).

- When the DE described in Storn and Price (1997) is considered as the COA, we use the algorithm $B_{2}$ introduced in Section 3.2 with $t_{1}=10$ and $t_{2}=1000$. This algorithm is denoted by DMA (DE MultiLayer Algorithm). The DE parameters used here are the following: the population size is set to 10 , the crossover operator is rand/1/exp, the crossover constant is 0.95 , the mutation parameter is 0.9 and the 
maximum number of iterations is 100. Those parameters have been determined experimentally. The Matlab implementation of the DE can be found here:

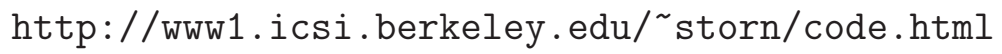

- When the CRS detailed in Hendrix et al. (2001) is considered as the COA, we use the algorithm $B_{2}$ introduced in Section 3.2 with $t_{1}=10$ and $t_{2}=1000$. This algorithm is denoted by CMA (CRS Multi-Layer Algorithm). The CRS parameters used here are the following: the population size is set to 60 , the number of trial points is set to the size of the problem, the maximum number of iterations is set to 300 and the rate of success test is 0.55 . These parameters have been determined experimentally.

Furthermore, at the end of the GMA, CMA and DMA, 10 iterations of the SD used in SMA1 are performed to improve the accuracy of the results.

Also, the performances of MLAs are compared with those of other metaheuristic methods available in the literature. We consider algorithms CGR and DTS whose implementation, parameters and results are presented in Hedar and Fukushima (2006) and Hirsch et al. (2010), respectively. We point out that there are other versions of Tabu Search and GRASP that may be superior for the problems tested (for example, particular choices of frequency-based memory have been found to significantly improve Tabu Search in various applications, see Bozkaya et al. (2003) and Glover (1997), and the hybridization of GRASP with Path Relinking has been found to significantly improve GRASP, see Piana et al. (2004) and Resende et al. (2010)). Furthermore, to see if applying the MLAs allows to improve the performances of the different COAs, we also solve the considered benchmark problems by considering the COAs alone with the following parameters:

- The SD and the HB are run starting from a random point in $\Omega$, with the same parameters as in SMA1 and HMA except $t_{0}=3000$.

- The GA is run with the same stochastic processes as GMA but with $N_{g}=1000, N_{p}=180, p_{c}=0.45$ and $p_{m}=0.15$. The stopping criterion considered here is explained below. These parameters have been identified in previous works (Ivorra et al., 2006, 2014). 
- The DE is run with the crossover operator set to rand/1/exp, the crossover constant set to 0.9 , the mutation parameters set to 0.5 , the population size set to 5 times the dimension of the benchmark problem and the maximum number of iterations set to 5000. These parameters were suggested in the literature for low dimensional problems (Storn and Price, 1997).

- The CRS is applied with a population size of 200, the number of trial points set to the size of the problem, the maximum number of iterations set to 3000 and the rate of success test set to 0.55 . These choices have been suggested in Hendrix et al. (2001).

Again, at the end of the GA, CRS and DR, 10 iterations of the SD used in SMA1 are carried out.

Following Hirsch et al. (2010), as the global minimum denoted by $h_{0}^{*}$, of the different benchmark problems is known, the stopping criterion is defined as

$$
\left|h_{0}^{*}-\tilde{h_{0}}\right| \leq \epsilon_{1}\left|h_{0}^{*}\right|+\epsilon_{2},
$$

where $\tilde{h_{0}}$ is the current solution of the algorithm, $\epsilon_{1}=10^{-4}$ and $\epsilon_{2}=10^{-6}$. The DE, DMA, CRS , CMA, GA and GMA are run with $\epsilon_{1}=10^{-2}$ and $\epsilon_{2}=10^{-3}$ and the SD performed at the end of those algorithms is executed with $\epsilon_{1}=10^{-4}$ and $\epsilon_{2}=10^{-6}$.

In addition to this stopping criterion we have limited the maximum number of functional evaluations to 50000 for each run (which can be considered as a high number, see Gomez et al. (2011); Ivorra et al. (2013, 2014); Muyl et al. (2004)). If at the end of the algorithm, (19) is not satisfied, we consider that the algorithm has failed to solve numerically the considered problem.

As specified in Section 3, we recall that the MLAs presented previously are adapted to non-negative functions (or with a known lower bound value). So, the benchmark functions $h_{0}$ with negative values have been modified adding a real number $C_{h_{0}}$ large enough to obtain a non-negative function. Here, in order to obtain a stopping criterion (19) comparable to the one used in Hedar and Fukushima (2006); Hirsch et al. (2010), we have considered $C_{h_{0}}=2\left|h_{0}^{*}\right|$.

Due to the stochastic aspect of the algorithms, each benchmark problem has been solved 100 times with each of the optimization algorithm. We define the success rate of an optimization algorithm by the percentage of runs satisfying the stopping criterion (19). 
In order to check the improvement of the MLA with respect to the COA alone, we have also computed the following improvement threshold (in \%), denoted by Imp and given by:

$$
\operatorname{Imp}(\mathbf{M L A})=100 \times \frac{\operatorname{Tev}(\mathbf{C O A})-\operatorname{Tev}(\mathbf{M L A})}{\operatorname{Tev}(\mathbf{C O A})},
$$

where $\operatorname{Tev}(\mathbf{A})$ is the total number of evaluations required by the algorithm $\mathbf{A}$ to solve all the benchmark problems (including runs that have failed to satisfy the stopping criterion (19)) and COA is the core optimization algorithm of MLA. Imp represents the computational effort reduction obtained when using the MLA instead of the COA.

All experiments have been performed on a Pentium I7 Quad-Core with 3.6 Ghz and $32 \mathrm{~Gb}$ of RAM and the algorithms have been implemented in a Matlab 2014 script.

Remark 3. The $\boldsymbol{M L A s}$ performs better if the minimum value $h_{0}^{*}=0$ (see Section 3.1.1). So, when $h_{0}^{*}$ is known (e.g., as said previously, in some inverse problems (Ivorra et al., 2014)), we can minimize $h_{0}-h_{0}^{*}$ instead of $h_{0}$ (Ivorra, 2006; Ivorra et al., 2014). However, in industrial applications, this information is generally not available (Carrasco et al., 2015; Gomez et al., 2011; Debiane et al., 2006; Ivorra et al., 2007, 2009; Muyl et al., 2004). Thus, we have decided not to use it. Furthermore, we deduce from the results presented in Section 4.1 .3 that this hypothesis is not mandatory as our methodology is efficient also in cases when $h_{0}^{*}$ is unknown.

Remark 4. The multi-layers linear search method was also applied alone to solve the considered benchmark problems. To do so, we considered $A_{0}\left(x_{0}\right)=$ $x_{0}$, and the 3-layers structure $A_{3}$ with $t_{0}=t_{1}=t_{2}=10$ and $t_{3}=10000$. However, this algorithm exhibited a success rate of $0 \%$ in all benchmark problems. Thus, the precision of this algorithm seems to be extremely low and the obtained results are not reported in the next Section 4.1.3. This indicates that the use of a $\boldsymbol{C O A}$ is necessary to generate an efficient $\boldsymbol{M L A}$.

\subsubsection{Results}

The average number of functional evaluations (considering only successful runs satisfying the stopping criterion (19)) needed by the optimization algorithms to solve the benchmark problems are shown in Table 1. The algorithms success rates are reported in Table 2. The value of Imp for each MLA is shown in Table 3. 


\begin{tabular}{|c|c|c|c|c|c|c|c|}
\hline Func. & DTS & CGR & SD & HB & GA & CRS & DE \\
\hline Bra & 212 & 10090 & 251 & 307 & 1304 & 2953 & 2347 \\
Eas & 223 & 5093 & - & 3600 & 40125 & 2877 & 3851 \\
G-P & 230 & 53 & 295 & 660 & 465 & 2429 & 1937 \\
Shu & 274 & 18608 & 120 & 1255 & 7748 & 9947 & 3049 \\
Hm3 & 438 & 1719 & 466 & 956 & 1119 & 1493 & 447 \\
Hm6 & 1787 & 29894 & 217 & 460 & 4418 & 2907 & 8456 \\
Rb2 & 254 & 23544 & 2275 & 1919 & 3918 & 6177 & 7952 \\
Rb5 & 1684 & 182520 & 3465 & 18287 & 43604 & 7927 & 41939 \\
Rb10 & 9037 & 725281 & 5096 & 25361 & 44557 & 43822 & 44156 \\
Sk5 & 819 & 9274 & 229 & 337 & 37328 & 5702 & 40032 \\
Sk7 & 812 & 11766 & 208 & 318 & 36046 & 3618 & 3479 \\
Sk10 & 6828 & 17612 & - & 401 & 40217 & 3540 & 2386 \\
Za5 & 1003 & 12467 & 268 & 600 & 24988 & 5384 & 40026 \\
Za10 & 4032 & 2297937 & 540 & 1770 & 40489 & 9004 & 40031 \\
\hline \hline Func. & SMA1 & SMA2 & SMA3 & HMA & GMA & CMA & DMA \\
\hline Bra & 215 & 140 & 130 & 489 & 252 & 2936 & 1821 \\
Eas & 2996 & 7085 & 7427 & 16504 & 3488 & 9801 & 1829 \\
G-P & 463 & 312 & 425 & 1292 & 439 & 3846 & 1054 \\
Shu & 416 & 4274 & 4125 & 12687 & 1270 & 9669 & 1557 \\
Hm3 & 564 & 347 & 415 & 1052 & 425 & 959 & 3613 \\
Hm6 & 766 & 369 & 591 & 5681 & 1054 & 5466 & 2972 \\
Rb2 & 1542 & 945 & 1210 & 2568 & 1675 & 4319 & 1353 \\
Rb5 & 4420 & 1777 & 1866 & 11513 & 43972 & 17351 & 10307 \\
Rb10 & 5612 & 2657 & 2545 & 21151 & 44828 & 42691 & 42930 \\
Sk5 & 3220 & 1123 & 1944 & 9540 & 6991 & 7453 & 6902 \\
Sk7 & 2648 & 936 & 1483 & 14780 & 4619 & 8343 & 4961 \\
Sk10 & 3336 & 3159 & 2416 & 6299 & 1637 & 8869 & 3653 \\
Za5 & 291 & 152 & 145 & 648 & 2674 & 7087 & 4106 \\
Za10 & 593 & 309 & 292 & 2110 & 20719 & 9860 & 17780 \\
\hline
\end{tabular}

Table 1: Average number (considering only the runs satisfying the stopping criterion (19)) of functional evaluations needed by the optimization algorithms described in Section 4.1.2 to solve the benchmark problems (Func.) presented in Section 4.1.1. 


\begin{tabular}{|c|c|c|c|c|c|c|c|}
\hline Func. & DTS & CGR & SD & HB & GA & CRS & DE \\
\hline Bra & 100 & 100 & 100 & 100 & 100 & 100 & 100 \\
Eas & 82 & 100 & 0 & 13 & 100 & 100 & 100 \\
G-P & 100 & 100 & 53 & 100 & 100 & 100 & 100 \\
Shu & 92 & 100 & 25 & 20 & 100 & 100 & 100 \\
Hm3 & 100 & 100 & 51 & 58 & 100 & 100 & 100 \\
Hm6 & 83 & 100 & 48 & 73 & 100 & 90 & 51 \\
Rb2 & 100 & 100 & 80 & 100 & 100 & 100 & 100 \\
Rb5 & 85 & 100 & 74 & 87 & 96 & 87 & 97 \\
Rb10 & 85 & 100 & 71 & 15 & 95 & 68 & 93 \\
Sk5 & 57 & 100 & 16 & 53 & 97 & 17 & 74 \\
Sk7 & 65 & 100 & 7 & 48 & 96 & 23 & 88 \\
Sk10 & 52 & 100 & 0 & 26 & 96 & 10 & 93 \\
Za5 & 100 & 100 & 100 & 100 & 100 & 100 & 100 \\
Za10 & 100 & 100 & 100 & 100 & 100 & 100 & 100 \\
\hline \hline Func. & SMA1 & SMA2 & SMA3 & HMA & GMA & CMA & DMA \\
\hline Bra & 100 & 100 & 100 & 100 & 100 & 100 & 100 \\
Eas & 12 & 100 & 100 & 48 & 100 & 100 & 100 \\
G-P & 100 & 100 & 100 & 100 & 100 & 100 & 100 \\
Shu & 47 & 100 & 100 & 91 & 100 & 100 & 100 \\
Hm3 & 100 & 100 & 100 & 100 & 100 & 100 & 100 \\
Hm6 & 100 & 100 & 100 & 95 & 100 & 100 & 49 \\
Rb2 & 95 & 100 & 100 & 100 & 100 & 100 & 100 \\
Rb5 & 100 & 100 & 100 & 97 & 92 & 100 & 95 \\
Rb10 & 100 & 100 & 100 & 64 & 81 & 100 & 92 \\
Sk5 & 66 & 100 & 100 & 66 & 96 & 100 & 97 \\
Sk7 & 37 & 100 & 100 & 57 & 98 & 100 & 98 \\
Sk10 & 30 & 100 & 100 & 55 & 97 & 94 & 95 \\
Za5 & 100 & 100 & 100 & 100 & 100 & 100 & 100 \\
Za10 & 100 & 100 & 100 & 100 & 100 & 100 & 100 \\
\hline
\end{tabular}

Table 2: Success rate (\%) of the optimization algorithms described in Section 4.1.2 when solving the benchmark problems (Func.) presented in Section 4.1.1. 


\begin{tabular}{|c|c|c|c|c|c|c|c|}
\hline MLA & SMA1 & SMA2 & SMA3 & HMA & GMA & CMA & DMA \\
\hline Imp & 50 & 85 & 93 & 33 & 58 & 41 & 50 \\
\hline
\end{tabular}

Table 3: Value of Imp (\%), defined by Equation (20), obtained when solving the benchmark problems presented in Section 4.2.1 with the following MLAs: SMA1, SMA2, SMA3, HMA, GMA, CMA and DMA.

Table 2 shows that $\mathbf{S D}$ and $\mathbf{H D}$ have the worst success rates for various benchmark functions. However, we also observe on this table that considering these algorithms as COAs used together with MLAs always improve their success rates. The mean number of evaluations required to satisfy the stopping criterion, presented in Table 1, is generally lower for a MLA than for its COA alone. In cases where the number of evaluations is greater for MLA than for its COA alone, we have to take into account that we only consider here the runs for which the algorithms satisfy the stopping criterion. This increases the computational effort of the MLA as its success rate is improved in comparison to its COA alone, and thus, the additional successful runs require a high number of functional evaluations. The advantage of using MLA can be also observed through the Imp values presented in Table 3. We can see that the improvement with MLA regarding the total number of evaluations required to solve all benchmark runs is increased from $33 \%$ up to 95\%. Those results indicate that the MLA method improves the efficiency of the COA for all these low dimensional problems.

Also, when analyzing the global efficiency of all proposed algorithms, the results indicate that SMA2 and SMA3 give the best performances. Indeed, both algorithms have a success percentage of 100 (as CGR) and are more efficient with respect to the number of functional evaluations, compared to all other methods. These results can be explained by the secant method chosen to perform the line search: as specified in Section 3.1.1, this method is well adapted for quick search and allows to have a rapid idea of the functional behavior (for instance, the monotonicity of the function in a given direction). Once this is detected the SD performs a local optimization. The Shubert (Shu) and Easom (Eas) benchmark cases are the two functions which require the largest numbers of evaluations for SMA2 and SMA3. This is because both functions have several local minima with small attraction basins and none is coercive (a function $f(x)$ is coercive if $\|x\| \rightarrow+\infty$ implies $f(x) \rightarrow+\infty$ ). This makes the global minimum attraction basin difficult to find using the 
secant method. As a conclusion, at this point SMA2 and SMA3 seem to represent the best choices.

When focusing on each MLA, SMA1 presents the lowest success rates of all MLAs. This is due to fact that in SMA1 the search for a suitable initial condition is only a 1D search while SMA2 and SMA3 introduce multidirectional explorations. With HMA the number of functional evaluations is higher and the success percentage is lower than with SMA2 and SMA3. This is because HB permits for better explorations of the admissible space due to its inertial features but too much inertia prevent from convergence. Finally GMA, CMA and DMA present interesting characteristics. Their success rates are generally equivalent to the GA and better than DTS, CRS and DE. Furthermore, depending on the cases, they generally require a lower number of functional evaluations than GA, CRS or DE alone. Therefore, these algorithms are good substitutes to classical evolutionary algorithms in cases where the gradient of the functional is difficult to access.

Remark 5. In addition to the low dimensional benchmark results presented above, $\boldsymbol{S M A 2}$ and $\boldsymbol{G M A}$ have been applied to the solution of several low dimensional design problems involving computationally expensive cost functions with often several local minima. The application domains concern: structural design (Carrasco et al., 2015, 2012), oil skimmer trajectory optimization (Gomez et al., 2011), synthesis of optical fiber (Ivorra et al., 2014), optimization of microfluidic mixers (Ivorra et al., 2013, 2006), temperature and pollution control in flames (Debiane et al., 2006), credit portfolio risk management (Ivorra et al., 2009), control of the solution for a PDE (Ivorra et al., 2007), optimization of the shape of coastal structures (Isebe et al., 2008).

\subsection{High dimensional benchmark problems}

\subsubsection{Considered benchmark problems}

We now consider the following benchmark box-constrained optimization problems in dimension $D$ (Floudas and Pardalos, 1999): Griewank (GrD), Rosenbrock (RbD) and Zakharov (ZaD). Following Li et al. (2013) D is set to $50,100,500$ and 1000 .

\subsubsection{Considered algorithms and parameters}

To solve the benchmark problems introduced in Section 4.2.1, we only consider those algorithms presented in Section 4.1.2 which have well performed in Section 4.1.3. More precisely, we only consider SD, GA, DE, 
CRS, SMA3, GMA, CMA and DMA. SMA1, HB and HMA are omitted due to their poor performances there. Also SMA2 is not reported as it has similar behavior than SMA3.

The differences when comparing the parameters with those used in Section 4.1.3 are:

- DE: the population size is set to 60 and the maximum number of iteration is set to 3000, as proposed in Gardeux et al. (2011).

- CRS: the population size is set to 400 (see, Hendrix et al. (2001)).

- SD: $t_{0}=20000$.

- GA: the population size is set to 250 .

- SMA3: $t_{0}=300$.

- GMA: The population size is set to 100 and the maximum number of iterations to 20 .

- CMA: The population size is set to 50 and the maximum number of iterations is set to 1000 .

- DMA: The population size is set to 60 and the maximum number of iterations is set to 100 .

The parameters for SD, SMA3, GMA, CMA and DMA are determined experimentally. The same stopping criterion (19) used in Section 4.1.2 is considered here. The maximum number of functional evaluations is set to 150000. Each experiment is repeated 100 times.

\subsubsection{Results}

The average number of functional evaluations (considering only successful runs satisfying the stopping criterion (19)) needed by the optimization algorithms to solve the benchmark problems are shown in Table 4. The success rate of the algorithms are reported in Table 5. Furthermore, as suggested in Li et al. (2013); Gardeux et al. (2011), we also report in Table 6 the mean final value of the cost function returned by each optimization algorithm for each benchmark problem over the 100 runs. Finally, the Imp values (20) of SMA3, GMA, CMA and DMA are shown in Table 7. 
From Table 5, we see that the MLAs exhibit better success rates than their respective COAs. Furthermore, as observed in Table 4, if the stopping criterion (19) is satisfied, the average number of cost function evaluations is generally lower for MLAs than for their associated COAs. Those results indicate that, as in the low dimensional cases, MLAs seem to improve the efficiency of the considered COA. However, for these high dimensional cases the improvement is not as important as for the low dimensional cases. Indeed, we can see in Table 7 that the Imp values of considered MLAs are between $2 \%$ and $30 \%$ (instead of $33 \%$ and $95 \%$ as in the low dimensional cases). Furthermore, we see that when the cost function is difficult to minimize (such as, Rb-1000 or Za50-Za1000), MLAs do not improve the success rate, which stays at $0 \%$. Thus, a COA well adapted to the considered optimization problem should be selected in order to create an efficient MLA.

Focusing on the global performances of the algorithms, results in Table 6 seem to indicate that the SMA3 gives the best results. In particular, for the Za50-Za1000 cases, SMA3 performs much better. For benchmark functions Gr50-Gr1000 and Rb50-Rb1000 all algorithms give similar solutions. When the gradient evaluation is not possible (as in some industrial problems where the cost function is computed by using a black-blox software, see Ivorra et al. (2006, 2013)) GMA, CMA and DMA present good alternatives to SMA3.

From a general point of view, the results reported in Table 6 are similar to other studies in the literature including different algorithms solving high dimensional cases (see, for instance, Gardeux et al. (2011)): the solution of the Griewank case can be approximated with a good precision whereas the minimum of the Rosenbrock function is difficult to evaluate.

\section{Conclusions}

A new multi-layer line search method, denoted by MLA, has been developed. This metaheuristic algorithm solves a sub-optimization problem in order to improve the initialization of existing optimization procedures considered as core optimization algorithm (COA). A particular implementation of the approach, well suited for minimizing non-negative functions, has been presented and coupled with various COAs: Steepest Descent, Heavy-Ball, Genetic, Differential Evolution and Controlled Random Search algorithms. The MLAs have been validated on various benchmark problems from low (with dimension less than 10) to high (with dimension up to 1000) dimen- 


\begin{tabular}{|c|c|c|c|c|}
\hline Func. & SD & GA & CRS & DE \\
\hline Gr50 & 7333 & 54629 & 96406 & 3597 \\
Gr100 & 18540 & 80117 & 113500 & 90766 \\
Gr500 & - & 125964 & 125500 & 120117 \\
Gr1000 & - & 146934 & 143137 & 147395 \\
Rb50 & 108768 & 125781 & 119018 & 130804 \\
Rb100 & - & 135409 & 125409 & 137854 \\
Rb500 & - & 149635 & 135184 & - \\
Rb1000 & - & - & - & - \\
Za50 & 3540 & - & - & - \\
Za100 & - & - & - & - \\
Za500 & - & - & - & - \\
Za1000 & - & - & - & - \\
\hline Func. & SMA3 & GMA & CMA & DMA \\
\hline Gr50 & 3409 & 40792 & 89347 & 2882 \\
Gr100 & 9749 & 70562 & 100137 & 5459 \\
Gr500 & 98353 & 109766 & 123830 & 73413 \\
Gr1000 & 141378 & 135028 & 142552 & 105185 \\
Rb50 & 7999 & 119781 & 117781 & 127612 \\
Rb100 & 62415 & 122796 & 122796 & 134453 \\
Rb500 & 78749 & 146002 & 125409 & - \\
Rb1000 & - & - & - & - \\
Za50 & 4858 & - & - & 56578 \\
Za100 & - & - & - & - \\
Za500 & - & - & - & - \\
Za1000 & - & - & - & - \\
\hline
\end{tabular}

Table 4: Average number (considering only the runs satisfying the stopping criterion (19)) of functional evaluations needed by the optimization algorithms described in Section 4.2.2 to solve the benchmark problems (Func.) presented in Section 4.2.1. 


\begin{tabular}{|c|c|c|c|c|c|c|c|c|}
\hline Func. & SD & GA & CRS & DE & SMA3 & GMA & CMA & DMA \\
\hline Gr50 & 100 & 100 & 100 & 100 & 100 & 100 & 100 & 100 \\
Gr100 & 100 & 100 & 100 & 100 & 100 & 100 & 100 & 100 \\
Gr500 & 0 & 100 & 100 & 100 & 100 & 100 & 100 & 100 \\
Gr1000 & 0 & 100 & 100 & 100 & 100 & 100 & 100 & 100 \\
Rb50 & 15 & 95 & 83 & 81 & 100 & 97 & 89 & 85 \\
Rb100 & 0 & 79 & 73 & 68 & 86 & 82 & 76 & 75 \\
Rb500 & 0 & 69 & 27 & 0 & 47 & 72 & 39 & 0 \\
Rb1000 & 0 & 0 & 0 & 0 & 0 & 0 & 0 & 0 \\
Za50 & 17 & 0 & 0 & 0 & 100 & 0 & 0 & 73 \\
Za100 & 0 & 0 & 0 & 0 & 0 & 0 & 0 & 0 \\
Za500 & 0 & 0 & 0 & 0 & 0 & 0 & 0 & 0 \\
Za1000 & 0 & 0 & 0 & 0 & 0 & 0 & 0 & 0 \\
\hline
\end{tabular}

Table 5: Success rate (\%) of the optimization algorithms described in Section 4.2 .2 when solving the benchmark problems (Func.) presented in Section 4.2.1.

sional cases. The numerical results seem to indicate that our methodology improves the performances of the COAs. The general conclusion is that MLAs with COAs given by gradient descent algorithms (SMA2 and SMA3) and evolutionary algorithms (GMA, CMA and DMA) should be preferred. These latter are good alternatives to other metaheuristic algorithms such as DTS, DE, CRS and CGR.

Our current effort is on parallel MLAs (Gomez et al., 2011).

A Matlab version of some of the algorithms presented in this paper has been implemented in the free optimization package "Global Optimization Platform", which can be downloaded at

http://www.mat.ucm.es/momat/software.htm

\section{Acknowledgements}

This work has been supported by the Spanish Ministry of Economy and Competitiveness under projects MTM2011-22658; the Junta de Andalucía; the European Regional Development Fund (ERDF) through projectsP12TIC301; the research group MOMAT (Ref. 910480) supported by "Banco Santander" and the "Universidad Complutense de Madrid". We would like to thank Dr. Juana Lopez Redondo and Prof. Pilar M. Ortigosa, from the 


\begin{tabular}{|c|c|c|c|c|}
\hline Func. & SD & GA & CRS & DE \\
\hline Gr50 & $10^{-6}$ & $10^{-6}$ & $10^{-6}$ & $10^{-6}$ \\
Gr100 & $10^{-6}$ & $10^{-6}$ & $10^{-6}$ & $10^{-6}$ \\
Gr500 & $10^{-4}$ & $10^{-6}$ & $10^{-6}$ & $10^{-6}$ \\
Gr1000 & $10^{-2}$ & $10^{-6}$ & $10^{-6}$ & $10^{-6}$ \\
Rb50 & $3 \times 10^{-2}$ & $5 \times 10^{-3}$ & $9 \times 10^{-3}$ & $7 \times 10^{-3}$ \\
Rb100 & 59 & 5.8 & 7.5 & 8.9 \\
Rb500 & 96 & 20 & 47 & 68 \\
Rb1000 & 219 & 139 & 147 & 168 \\
Za50 & 633 & 37 & 1800 & 1228 \\
Za100 & 2664 & 38845 & 28448 & 83252 \\
Za500 & 98740 & 40021 & 79441 & 97333 \\
Za1000 & $3.5 \times 10^{5}$ & 83642 & $1.2 \times 10^{5}$ & $1.6 \times 10^{5}$ \\
\hline Func. & SMA3 & GMA & CMA & DMA \\
\hline Gr50 & $10^{-6}$ & $10^{-6}$ & $10^{-6}$ & $10^{-6}$ \\
Gr100 & $10^{-6}$ & $10^{-6}$ & $10^{-6}$ & $10^{-6}$ \\
Gr500 & $10^{-6}$ & $10^{-6}$ & $10^{-6}$ & $10^{-6}$ \\
Gr1000 & $10^{-6}$ & $10^{-6}$ & $10^{-6}$ & $10^{-6}$ \\
Rb50 & $10^{-6}$ & $3 \times 10^{-3}$ & $7 \times 10^{-3}$ & $6 \times 10^{-3}$ \\
Rb100 & 3.9 & 5.0 & 3.1 & 4.2 \\
Rb500 & 162 & 30 & 39 & 63 \\
Rb1000 & 195 & 125 & 136 & 145 \\
Za50 & $10^{-6}$ & 1 & 1512 & 0.5 \\
Za100 & 24 & 26159 & 13052 & 22784 \\
Za500 & 403 & 30897 & 73362 & 83252 \\
Za1000 & 7812 & 67423 & $1.1 \times 10^{5}$ & $1.3 \times 10^{5}$ \\
\hline
\end{tabular}

Table 6: Mean value of the cost function obtained for the benchmark problems (Func.) presented in Section 4.2.1 with the optimization algorithms described in Section 4.2.2.

\begin{tabular}{|c|c|c|c|c|}
\hline MLA & SMA3 & GMA & CMA & DMA \\
\hline Imp & 30 & 5 & 2 & 16 \\
\hline
\end{tabular}

Table 7: Value of Imp (\%), defined by Equation (20), obtained when solving the benchmark problems presented in Section 4.2.1 with the following MLA: SMA3, GMA, CMA and DMA. 
"Universidad de Almería", for providing us with a Matlab implementation of the Controlled Random Search algorithm.

Ashlock, D. (2010). Evolutionary Computation for Modeling and Optimization. Springer Publishing Company, Incorporated, 1st edition.

Attouch, H., Goudou, X., and Redont, P. (2000). The heavy ball with friction method, i. the continuous dynamical system: Global exploration of the local minima of a real-valued function by asymptotic analysis of a dissipative dynamical system. Communications in Contemporary Mathematics, 02(01):1-34.

Bozkaya, B., Erkut, E., and Laporte, G. (2003). A tabu search heuristic and adaptive memory procedure for political districting. European Journal of Operational Research, 144(1):12 - 26.

Carrasco, M., Ivorra, B., and Ramos, A. M. (2012). A variance-expected compliance model for structural optimization. Journal of Optimization Theory and Applications, 152(1):136-151.

Carrasco, M., Ivorra, B., and Ramos, A. M. (2015). Stochastic topology design optimization for continuous elastic materials. Computer Methods in Applied Mechanics and Engineering.

Debiane, L., Ivorra, B., Mohammadi, B., Nicoud, F., Poinsot, T., Ern, A., and Pitsch, H. (2006). A low-complexity global optimization algorithm for temperature and pollution control in flames with complex chemistry. International Journal of Computational Fluid Dynamics, 20(2):93-98.

Floudas, C. and Pardalos, P. (1999). Handbook of test problems in local and global optimization. Nonconvex optimization and its applications. Kluwer Academic Publishers.

Gardeux, V., Chelouah, R., Siarry, P., and Glover, F. (2009). Unidimensional search for solving continuous high-dimensional optimization problems. In Intelligent Systems Design and Applications, 2009. ISDA '09. Ninth International Conference on, pages 1096-1101.

Gardeux, V., Chelouah, R., Siarry, P., and Glover, F. (2011). Em323: a line search based algorithm for solving high-dimensional continuous non-linear optimization problems. Soft Computing, 15(11):2275-2285. 
Glover, F. (1997). Tabu search and adaptive memory programming advances, applications and challenges. In Barr, R., Helgason, R., and Kennington, J., editors, Interfaces in Computer Science and Operations Research, volume 7 of Operations Research/Computer Science Interfaces Series, pages 1-75. Springer US.

Glover, F. (2010). The 3-2-3, stratified split and nested interval line search algorithms. Research report, OptTek Systems, Boulder, CO.

Goldberg, D. E. (1989). Genetic Algorithms in Search, Optimization and Machine Learning. Addison-Wesley Longman Publishing Co., Inc., Boston, MA, USA, 1st edition.

Gomez, S., Ivorra, B., and Ramos, A. M. (2011). Optimization of a pumping ship trajectory to clean oil contamination in the open sea. Mathematical and Computer Modelling, 54(12):477 - 489.

Gomez, S. and Levy, A. (1982). The tunnelling method for solving the constrained global optimization problem with several non-connected feasible regions. In Hennart, J., editor, Numerical Analysis, volume 909 of Lecture Notes in Mathematics, pages 34-47. Springer Berlin Heidelberg.

Gonalves, J. F., de Magalhes Mendes, J. J., and Resende, M. G. C. (2002). A hybrid genetic algorithm for the job shop scheduling problem. European Journal of Operational Research, 167:2005.

Grosan, C. and Abraham, A. (2007). Hybrid line search for multiobjective optimization. In Perrott, R., Chapman, B., Subhlok, J., de Mello, R., and Yang, L., editors, High Performance Computing and Communications, volume 4782 of Lecture Notes in Computer Science, pages 62-73. Springer Berlin Heidelberg.

Hale, J. (2009). Ordinary Differential Equations. Dover Books on Mathematics Series. Dover Publications.

Hedar, A.-R. and Fukushima, M. (2006). Tabu search directed by direct search methods for nonlinear global optimization. European Journal of Operational Research, 170(2):329 - 349.

Hendrix, E., Ortigosa, P., and Garca, I. (2001). On success rates for controlled random search. Journal of Global Optimization, 21(3):239-263. 
Hirsch, M., Pardalos, P., and Resende, M. (2010). Speeding up continuous GRASP. European Journal of Operational Research, 205(3):507 - 521.

Isebe, D., Azerad, P., Bouchette, F., Ivorra, B., and Mohammadi, B. (2008). Shape optimization of geotextile tubes for sandy beach protection. International Journal for Numerical Methods in Engineering, 74(8):1262-1277.

Ivorra, B. (2006). Optimisation globale semi-deterministe et applications industrielles. ANRT-Grenoble, Reference: 06/MON2/0061.

Ivorra, B., Hertzog, D. E., Mohammadi, B., and Santiago, J. G. (2006). Semideterministic and genetic algorithms for global optimization of microfluidic protein-folding devices. International Journal for Numerical Methods in Engineering, 66(2):319-333.

Ivorra, B., Mohammadi, B., and Ramos, A. M. (2009). Optimization strategies in credit portfolio management. Journal of Global Optimization, 43(2$3): 415-427$.

Ivorra, B., Mohammadi, B., and Ramos, A. M. (2014). Design of code division multiple access filters based on sampled fiber bragg grating by using global optimization algorithms. Optimization and Engineering, 15(3):677695.

Ivorra, B., Ramos, A. M., and Mohammadi, B. (2007). Semideterministic global optimization method: Application to a control problem of the burgers equation. Journal of Optimization Theory and Applications, 135(3):549-561.

Ivorra, B., Redondo, J. L., Santiago, J. G., Ortigosa, P. M., and Ramos, A. M. (2013). Two- and three-dimensional modeling and optimization applied to the design of a fast hydrodynamic focusing microfluidic mixer for protein folding. Physics of Fluids (1994-present), 25(3):-.

Lamghari, A. and Dimitrakopoulos, R. (2012). A diversified tabu search approach for the open-pit mine production scheduling problem with metal uncertainty. European Journal of Operational Research, 222(3):642 - 652.

Levy, A. and Gomez., S. (1985). The tunneling method applied to global optimization. In Numerical Optimization 1984: Proceedings of the SIAM 
Conference on Numerical Optimization, Boulder, Colorado, June 12-14, 1984. Proceedings in Applied Mathematics Series. SIAM.

Li, X., Engelbrecht, A., and Epitropakis, M. G. (2013). Benchmark functions for cec'2013 special session and competition on niching methods for multimodal function optimization. 2013 IEEE Congress on Evolutionary Computation.

Luenberger, D. and Ye, Y. (2008). Linear and Nonlinear Programming. International Series in Operations Research \& Management Science. Springer.

Mart, R., Campos, V., Resende, M. G., and Duarte, A. (2015). Multiobjective GRASP with path relinking. European Journal of Operational Research, 240(1):54 - 71 .

Mohammadi, B. and Saïac, J. (2003). Pratique de la simulation numérique. Conception: Industrie et Technologies. Dunod.

Muyl, F., Dumas, L., and Herbert, V. (2004). Hybrid method for aerodynamic shape optimization in automotive industry. Computers and Fluids, $33(5)$.

Piana, E., Plana, I., Campos, V., and Martí, R. (2004). GRASP and path relinking for the matrix bandwidth minimization. European Journal of Operational Research, 153(1):200 - 210. Timetabling and Rostering.

Polyak, B. (2007). Newtons method and its use in optimization. European Journal of Operational Research, 181(3):1086 - 1096.

Price, K., Storn, R. M., and Lampinen, J. A. (2005). Differential Evolution: A Practical Approach to Global Optimization (Natural Computing Series). Springer-Verlag New York, Inc., Secaucus, NJ, USA.

Price, W. (1983). Global optimization by controlled random search. Journal of Optimization Theory and Applications, 40(3):333-348.

Redondo, J. L., Fernández, J., García, I., and Ortigosa, P. M. (2009). Solving the multiple competitive facilities location and design problem on the plane. Evol. Comput., 17(1):21-53. 
Resende, M., Ribeiro, C., Glover, F., and Mart, R. (2010). Scatter search and path-relinking: Fundamentals, advances, and applications. In Gendreau, M. and Potvin, J.-Y., editors, Handbook of Metaheuristics, volume 146 of International Series in Operations Research 85 Management Science, pages 87-107. Springer US.

Rocha, M. and Neves, J. (1999). Preventing premature convergence to local optima in genetic algorithms via random offspring generation. In Imam, I., Kodratoff, Y., El-Dessouki, A., and Ali, M., editors, Multiple Approaches to Intelligent Systems, volume 1611 of Lecture Notes in Computer Science, pages 127-136. Springer Berlin Heidelberg.

Storn, R. and Price, K. (1997). Differential evolution a simple and efficient heuristic for global optimization over continuous spaces. Journal of Global Optimization, 11(4):341-359.

Verhulst, F. (1996). Nonlinear Differential Equations and Dynamical Systems. Hochschultext / Universitext. Springer Berlin Heidelberg.

Vieira, D. A. G. and Lisboa, A. C. (2014). Line search methods with guaranteed asymptotical convergence to an improving local optimum of multimodal functions. European Journal of Operational Research, 235(1):38 46. 